\title{
Central limit theorem for a fractional stochastic heat equation with spatially correlated noise
}

\section{Yumeng $\mathrm{Li}^{1 *}$}

\section{"Correspondence:}

liyumeng@mail.ustc.edu.cn

'School of Statistics and

Mathematics, Zhongnan University

of Economics and Law, Wuhan,

P.R. China

\begin{abstract}
In this paper, we study the central limit theorem for a perturbed stochastic heat equation in the whole space $\mathbb{R}^{d}, d \geq 1$. This equation is driven by a Gaussian noise, which is white in time and correlated in space, and the differential operator is a fractional derivative operator. Burkholder's inequality plays an important role in the proof.

MSC: $60 \mathrm{H} 15 ; 60 \mathrm{~F} 05 ; 60 \mathrm{~F} 10$

Keywords: Fractional derivative operator; Stochastic heat equation; Central limit
\end{abstract} theorem

\section{Introduction}

In this paper, we study the central limit theorem for a fractional stochastic heat equation in spatial dimension $\mathbb{R}^{d}$ driven by a spatially correlated noise:

$$
\left\{\begin{array}{l}
\frac{\partial u^{\varepsilon}}{\partial t}(t, x)=\mathcal{D}_{\underline{\delta}}^{\alpha} u^{\varepsilon}(t, x)+b\left(u^{\varepsilon}(t, x)\right)+\sqrt{\varepsilon} \sigma\left(u^{\varepsilon}(t, x)\right) \dot{F}(t, x), \\
u^{\varepsilon}(0, x)=0
\end{array}\right.
$$

where $\varepsilon>0,(t, x) \in[0, T] \times \mathbb{R}^{d}, d \geq 1, \underline{\alpha}=\left(\alpha_{1}, \ldots, \alpha_{d}\right), \underline{\delta}=\left(\delta_{1}, \ldots, \delta_{d}\right)$, and we will assume that $\left.\left.\alpha_{i} \in\right] 0,2\right] \backslash\{1\}$ and $\left|\delta_{i}\right| \leq \min \left\{\alpha_{i}, 2-\delta_{i}\right\}, i=1, \ldots, d, \dot{F}$ is the "formal" derivative of the Gaussian perturbation and $\mathcal{D}_{\underline{\delta}}^{\underline{\alpha}}$ denotes a non-local fractional differential operator on $\mathbb{R}^{d}$ defined by $\mathcal{D}_{\underline{\delta}}^{\alpha}:=\sum_{i=1}^{d} D_{\delta_{i}}^{\alpha_{i}}$. Here, $D_{\delta_{i}}^{\alpha_{i}}$ denotes the fractional differential derivative with respect to the $i$ th coordinate defined via its Fourier transform $\mathcal{F}$ by

$$
\mathcal{F}\left(\mathcal{D}_{\underline{\delta}}^{\underline{\alpha}} \phi\right)(\xi)=-|\xi|^{\alpha_{i}} \exp \left(-l \delta_{i} \frac{\pi}{2} \operatorname{sgn} \xi\right) \mathcal{F}(\phi)(\xi)
$$

with $\iota^{2}+1=0$. The noise $F(t, x)$ is a martingale measure in the sense of Walsh [12] and Dalang [4]. From now on, we shall refer to Eq. (1) as $E q_{\delta, \varepsilon}^{\alpha}(d, b, \sigma)$. See Sect. 2 for details.

(c) The Author(s) 2020. This article is licensed under a Creative Commons Attribution 4.0 International License, which permits use, sharing, adaptation, distribution and reproduction in any medium or format, as long as you give appropriate credit to the original author(s) and the source, provide a link to the Creative Commons licence, and indicate if changes were made. The images or other third party material in this article are included in the article's Creative Commons licence, unless indicated otherwise in a credit line to the material. If material is not included in the article's Creative Commons licence and your intended use is not permitted by statutory regulation or exceeds the permitted use, you will need to obtain permission directly from the copyright holder. To view a copy of this licence, visit http://creativecommons.org/licenses/by/4.0/. 
As the parameter $\varepsilon$ tends to zero, the solutions $u^{\varepsilon}$ of (1) will tend to the solution of the deterministic equation defined by

$$
\left\{\begin{array}{l}
\frac{\partial u^{0}}{\partial t}(t, x)=\mathcal{D}_{\underline{\underline{\delta}}}^{\alpha} u^{0}(t, x)+b\left(u^{0}(t, x)\right), \\
u^{0}(0, x)=0 .
\end{array}\right.
$$

It is always interesting to investigate deviations of $u^{\varepsilon}$ from $u^{0}$, as $\varepsilon$ decreases to 0 , that is, the asymptotic behavior of the trajectory

$$
X^{\varepsilon}(t, x):=\frac{1}{\sqrt{\varepsilon} \lambda(\varepsilon)}\left(u^{\varepsilon}-u^{0}\right)(t, x), \quad(t, x) \in[0, T] \times \mathbb{R}^{d},
$$

where $\lambda(\varepsilon)$ is some deviation scale which strongly influences the asymptotic behavior of $X^{\varepsilon}$ (see Freidlin and Wentzell [8]):

- The case $\lambda(\varepsilon)=1 / \sqrt{\varepsilon}$ provides some large deviation estimates. El Mellali and Mellouk [7] proved that the law of the solution $u^{\varepsilon}$ satisfies a large deviation principle.

- When the deviation scale satisfies

$$
\lambda(\varepsilon) \rightarrow+\infty, \quad \sqrt{\varepsilon} \lambda(\varepsilon) \rightarrow 0 \quad \text { as } \varepsilon \rightarrow 0,
$$

it is the moderate deviations. Li et al. [11] proved that $\frac{1}{\sqrt{\varepsilon} \lambda(\varepsilon)}\left(u^{\varepsilon}-u^{0}\right)$ satisfies a moderate deviation principle by the weak convergence method.

- When the deviation scale satisfies $\lambda(\varepsilon)=1$, we are in the domain of the central limit theorem. In this paper, we prove that the process $\left(u^{\varepsilon}-u^{0}\right) / \sqrt{\varepsilon}$ converges to a random field.

The central limit theorem is a traditional topic in the theory of probability and statistics. Recently, the study of the central limit theorem for stochastic (partial) differential equation has been carried out, see e.g. [2, 9, 11, 13] etc.

The rest of this paper is organized as follows. In Sect. 2, the precise framework is stated. In Sect. 3, we state the central limit theorem and prove it by establishing some moment convergence results of SPDE.

Throughout the paper, $C_{p}$ is a positive constant depending on the parameter $p$, and $C, C_{1}, \ldots$ are constants depending on no specific parameter (except $T$ and the Lipschitz constants), whose value may be different from line to line by convention.

\section{Framework}

In this section, let us give the framework taken from Boulanba et al. [1], El Mellali and Mellouk [7].

\subsection{The operator $\mathcal{D}_{\delta}^{\alpha}$}

According to [6,10], $\bar{D}_{\delta}^{\alpha}$ can be represented for $1<\alpha<2$ by

$$
D_{\delta}^{\alpha}=\int_{-\infty}^{+\infty} \frac{\phi(x+y)-\phi(x)-y \phi^{\prime}(x)}{|y|^{1+\alpha}}\left(\kappa_{-}^{\delta} \mathbf{1}_{(-\infty, 0)}(y)+\kappa_{+}^{\delta} \mathbf{1}_{(0,+\infty)}(y)\right) d y,
$$

and for $0<\alpha<1$ by

$$
D_{\delta}^{\alpha}=\int_{-\infty}^{+\infty} \frac{\phi(x+y)-\phi(x)}{|y|^{1+\alpha}}\left(\kappa_{-}^{\delta} \mathbf{1}_{(-\infty, 0)}(y)+\kappa_{+}^{\delta} \mathbf{1}_{(0,+\infty)}(y)\right) d y,
$$


where $\kappa_{-}^{\delta}$ and $\kappa_{+}^{\delta}$ are two non-negative constants satisfying $\kappa_{-}^{\delta}+\kappa_{+}^{\delta}>0$ and $\phi$ is a smooth function for which the integral exists, and $\phi^{\prime}$ stands for its derivative.

Let $G_{\alpha, \delta}(t, x)$ denote the fundamental solution of the equation $E q_{\delta, 1}^{\alpha}(1,0,0)$, that is, the unique solution of the Cauchy problem

$$
\left\{\begin{array}{l}
\frac{\partial u}{\partial t}(t, x)=D_{\delta}^{\alpha} u(t, x), \\
u(0, x)=\delta_{0}(x), \quad t>0, x \in \mathbb{R}
\end{array}\right.
$$

where $\delta_{0}$ is the Dirac distribution. Using Fourier's calculus, one gets

$$
G_{\alpha, \delta}(t, x)=\frac{1}{2 \pi} \int_{-\infty}^{\infty} \exp \left(-l z x-t|z|^{\alpha} \exp \left(-\imath \delta \frac{\pi}{2} \operatorname{sgn}(z)\right)\right) d z .
$$

Here, $\alpha \in] 0,2]$ and $|\delta| \leq \min \{\alpha, 2-\alpha\}$.

For higher dimension $d \geq 1$ and any multi index $\underline{\alpha}=\left(\alpha_{1}, \ldots, \alpha_{d}\right)$ and $\underline{\delta}=\left(\delta_{1}, \ldots, \delta_{d}\right)$, let $\mathbf{G}_{\underline{\alpha}, \underline{\delta}}(t, x)$ be the Green function of the deterministic equation $E q_{\underline{\delta}}^{\underline{\alpha}}(d, 0,0)$. Clearly,

$$
\begin{aligned}
\mathbf{G}_{\underline{\alpha}, \underline{\delta}}(t, x) & =\prod_{i=1}^{d} G_{\alpha_{i}, \delta_{i}}\left(t, x_{i}\right) \\
& =\frac{1}{(2 \pi)^{d}} \int_{\mathbb{R}^{d}} \exp \left(-\imath\langle\xi, x\rangle-t \sum_{i=1}^{d}\left|\xi_{i}\right|^{\alpha_{i}} \exp \left(-l \delta_{i} \frac{\pi}{2} \operatorname{sgn}\left(\xi_{i}\right)\right)\right) d \xi,
\end{aligned}
$$

where $\langle\cdot, \cdot\rangle$ stands for the inner product in $\mathbb{R}^{d}$.

\subsection{The driving noise $F$}

Let $\mathcal{S}\left(\mathbb{R}^{d+1}\right)$ be the space of Schwartz test functions. On a complete probability space $(\Omega, \mathcal{G}, \mathbb{P})$, the noise $F=\left\{F(\phi), \phi \in \mathcal{S}\left(\mathbb{R}^{d+1}\right)\right\}$ is assumed to be an $L^{2}(\Omega, \mathcal{G}, \mathbb{P})$-valued Gaussian process with mean zero and covariance functional given by

$$
J(\varphi, \psi):=\mathbb{E}[F(\phi) F(\psi)]=\int_{\mathbb{R}_{+}} d s \int_{\mathbb{R}^{d}}(\phi(s, \star) * \tilde{\psi}(s, \star))(x) \Gamma(d x) d s, \quad \phi, \psi \in \mathcal{S}\left(\mathbb{R}^{d+1}\right),
$$

where $\tilde{\psi}(s, x):=\psi(s,-x)$ and $\Gamma$ is a non-negative and non-negative definite tempered measure, therefore symmetric. The symbol $*$ denotes the convolution product and $\star$ stands for the spatial variable.

Let $\mu$ be the spectral measure of $\Gamma$, which is a tempered measure, that is, $\mu=\mathcal{F}^{-1}(\Gamma)$, and this gives

$$
J(\phi, \psi)=\int_{\mathbb{R}_{+}} d s \int_{\mathbb{R}^{d}} \mu(d \xi) \mathcal{F} \phi(s, \star)(\xi) \overline{\mathcal{F} \psi(s, \star)}(\xi),
$$

where $\bar{z}$ is the complex conjugate of $z$.

As in Dalang [4], the Gaussian process $F$ can be extended to a worthy martingale measure, in the sense of Walsh [12],

$$
M:=\left\{M_{t}(A), t \in \mathbb{R}_{+}, A \in \mathcal{B}_{b}\left(\mathbb{R}^{d}\right)\right\},
$$


where $\mathcal{B}_{b}\left(\mathbb{R}^{d}\right)$ denotes the collection of all bounded Borel measurable sets in $\mathbb{R}^{d}$. Let $\mathcal{G}_{t}$ be the completion of the $\sigma$-field generated by the random variables $\{F(s, A) ; 0 \leq s \leq t, A \in$ $\left.\mathcal{B}_{b}\left(\mathbb{R}^{d}\right)\right\}$.

Boulanba et al. [1] gave a rigorous meaning to the solution of equation $E q_{\underline{\delta}, \varepsilon}^{\alpha}(d, b, \sigma)$ by means of a joint measurable and $\mathcal{G}_{t}$-adapted process $\left\{u^{\varepsilon}(t, x) ;(t, x) \in \mathbb{R}_{+} \times \mathbb{R}^{d}\right\}$ satisfying, for each $t \geq 0$ and for almost all $x \in \mathbb{R}^{d}$, the following evolution equation:

$$
\begin{aligned}
u^{\varepsilon}(t, x)= & \sqrt{\varepsilon} \int_{0}^{t} \int_{\mathbb{R}^{d}} \mathbf{G}_{\underline{\alpha}, \underline{\delta}}(t-s, x-y) \sigma\left(u^{\varepsilon}(s, y)\right) F(d s d y) \\
& +\int_{0}^{t} d s \int_{\mathbb{R}^{d}} \mathbf{G}_{\underline{\alpha}, \underline{\delta}}(t-s, x-y) b\left(u^{\varepsilon}(s, y)\right) d y .
\end{aligned}
$$

In order to prove our result, we are going to give another equivalent approach to the solution of $E q_{\delta, \varepsilon}^{\alpha}(d, b, \sigma)$, see [5]. To start with, let us denote by $\mathcal{H}$ the Hilbert space obtained by the completion of $\mathcal{S}\left(\mathbb{R}^{d}\right)$ with the inner product

$$
\begin{aligned}
\langle\phi, \psi\rangle_{\mathcal{H}} & :=\int_{\mathbb{R}^{d}} \Gamma(d x)(\phi * \tilde{\psi})(x) \\
& =\int_{\mathbb{R}^{d}} \mu(d \xi) \mathcal{F} \phi(\xi) \overline{\mathcal{F} \psi}(\xi), \quad \phi, \psi \in \mathcal{S}\left(\mathbb{R}^{d}\right) .
\end{aligned}
$$

The norm induced by $\langle\cdot, \cdot\rangle_{\mathcal{H}}$ is denoted by $\|\cdot\|_{\mathcal{H}}$.

By Walsh's theory of the martingale measures [12], for $t \geq 0$ and $h \in \mathcal{H}$, the stochastic integral

$$
B_{t}(h)=\int_{0}^{t} \int_{\mathbb{R}^{d}} h(y) F(d s, d y)
$$

is well defined and the process $\left\{B_{t}(h) ; t \geq 0, h \in \mathcal{H}\right\}$ is a cylindrical Wiener process on $\mathcal{H}$. Let $\left\{e_{k}\right\}_{k \geq 1}$ be a complete orthonormal system of the Hilbert space $\mathcal{H}$, then

$$
\left\{B_{t}^{k}:=\int_{0}^{t} \int_{\mathbb{R}^{d}} e_{k}(y) F(d s, d y) ; k \geq 1\right\}
$$

defines a sequence of independent standard Wiener processes, and we have the following representation:

$$
B_{t}:=\sum_{k \geq 1} B_{t}^{k} e_{k}
$$

Let $\left\{\mathcal{F}_{t}\right\}_{t \in[0, T]}$ be the $\sigma$-field generated by the random variables $\left\{B_{s}^{k} ; s \in[0, t], k \geq 1\right\}$. We define the predictable $\sigma$-field in $\Omega \times[0, T]$ generated by the sets \{]$s, t] \times A ; A \in \mathcal{F}_{s}, 0 \leq s \leq$ $t \leq T\}$. In the following, we can define the stochastic integral with respect to cylindrical Wiener process $\left\{B_{t}(h)\right\}_{t \geq 0}$ (see e.g. [3] or [5]) of any predictable square-integrable process with values in $\mathcal{H}$ as follows:

$$
\int_{0}^{t} \int_{\mathbb{R}^{d}} g \cdot d B:=\sum_{k \geq 1} \int_{0}^{t}\left\langle g(s), e_{k}\right\rangle_{\mathcal{H}} d B_{s}^{k} .
$$


In the sequel, we shall consider the mild solution to equation $E q_{\underline{\delta}, \varepsilon}^{\frac{\alpha}{}}(d, b, \sigma)$ given by

$$
\begin{aligned}
u^{\varepsilon}(t, x)= & \sqrt{\varepsilon} \sum_{k \geq 1} \int_{0}^{t}\left\langle\mathbf{G}_{\underline{\alpha}, \underline{\delta}}(t-s, x-\cdot) \sigma\left(u^{\varepsilon}(s, \star)\right), e_{k}\right\rangle_{\mathcal{H}} d B_{s}^{k} \\
& +\int_{0}^{t}\left[\mathbf{G}_{\underline{\alpha}, \underline{\delta}}(t-s) * b\left(u^{\varepsilon}(s, \star)\right)\right](x) d s
\end{aligned}
$$

for any $t \in[0, T], x \in \mathbb{R}^{d}$.

\subsection{Existence, uniqueness, and Hölder regularity to equation}

For a multi-index $\underline{\alpha}=\left(\alpha_{1}, \ldots, \alpha_{d}\right)$ such that $\left.\left.\alpha_{i} \in\right] 0,2\right] \backslash\{1\}, i=1, \ldots, d$, and any $\xi \in \mathbb{R}^{d}$, let $S_{\underline{\alpha}}(\xi)=\sum_{i=1}^{d}\left|\xi_{i}\right|^{\alpha_{i}}$. Assume the following assumptions on the functions $\sigma, b$ and the measure $\mu$ :

(C): The functions $\sigma$ and $b$ are Lipschitz, that is, there exists some constant $L$ such that, for all $x, y \in \mathbb{R}$,

$$
\|\sigma(x)-\sigma(y)\| \leq L|x-y|, \quad|b(x)-b(y)| \leq L|x-y|
$$

$\left(H_{\eta}^{\alpha}\right):$ Let $\underline{\alpha}$ be as defined above and $\left.\left.\eta \in\right] 0,1\right]$, it holds that

$$
\int_{\mathbb{R}^{d}} \frac{\mu(d \xi)}{\left(1+S_{\underline{\alpha}}(\xi)\right)^{\eta}}<+\infty
$$

From Boulanba et al. [1], we know the following result.

Proposition 2.1 ([1, Theorem 3.1]) Under assumptions (C) and $\left(H_{\eta}^{\left.\frac{\alpha}{}\right)}\right.$, Eq. (8) admits a unique continuous solution $u^{\varepsilon}$, which satisfies

$$
\sup _{t \in[0, T], x \in \mathbb{R}^{d}} \mathbb{E}\left[\left|u^{\varepsilon}(t, x)\right|^{p}\right]<+\infty
$$

\subsection{Convergence of solutions}

The next result is concerned with the convergence of $u^{\varepsilon}$ as $\varepsilon \rightarrow 0$.

Proposition 2.2 Assume $(\mathrm{C})$ and $\left(H_{\eta}^{\frac{\alpha}{\eta}}\right)$. Then, for any $T>0$, there exists some constant $C(p, K, T)$ depending on $p, K, T$ such that

$$
\sup _{0 \leq t \leq T, x \in \mathbb{R}^{d}} \mathbb{E}\left[\left|u^{\varepsilon}(t, x)-u^{0}(t, x)\right|^{p}\right] \leq \varepsilon^{\frac{p}{2}} c(p, L, T) .
$$

Proof For any $(t, x) \in[0, T] \times \mathbb{R}^{d}$, we have

$$
\begin{aligned}
u^{\varepsilon}(t, x)-u^{0}(t, x)= & \sqrt{\varepsilon} \sum_{k \geq 1} \int_{0}^{t}\left\langle\mathbf{G}_{\underline{\alpha}, \underline{\delta}}(t-s, x-\cdot) \sigma\left(u^{\varepsilon}(s, \star)\right), e_{k}\right\rangle_{\mathcal{H}} d B_{s}^{k} \\
& +\int_{0}^{t}\left[\mathbf{G}_{\underline{\alpha}, \underline{\delta}}(t-s) *\left(b\left(u^{\varepsilon}(s, \star)\right)-b\left(u^{0}(s, \star)\right)\right)\right](x) d s \\
=: & A_{1}^{\varepsilon}+A_{2}^{\varepsilon} .
\end{aligned}
$$


Let

$$
\mathcal{J}(t):=\int_{\mathbb{R}^{d}} \mu(d \xi)\left|\mathcal{F} \mathbf{G}_{\underline{\alpha}, \underline{\delta}}(t)(\xi)\right|^{2} .
$$

For the first term $A_{1}^{\varepsilon}$, by Burkholder's inequality, the Lipschitz property of $\sigma$, and (10), we have that, for any $(t, x) \in[0, T] \times \mathbb{R}^{d}, p \geq 2$,

$$
\begin{aligned}
\mathbb{E}\left[\left|A_{1}^{\varepsilon}(t, x)\right|^{p}\right] & \leq \varepsilon^{\frac{p}{2}} c(p) \mathbb{E}\left[\left|\int_{0}^{t}\left\|\mathbf{G}_{\underline{\alpha}, \underline{\underline{\delta}}}(t-s, x-\star) \sigma\left(u^{\varepsilon}(s, \star)\right)\right\|_{\mathcal{H}}^{2} d s\right|^{\frac{p}{2}}\right] \\
& \leq \varepsilon^{\frac{p}{2}} c(p, T) \mathbb{E}\left[\int_{0}^{t}\left\|\mathbf{G}_{\underline{\alpha}, \underline{ }}(t-s, x-\star) \sigma\left(u^{\varepsilon}(s, \star)\right)\right\|_{\mathcal{H}}^{p} d s\right] \\
& \leq \varepsilon c(p, L, T) \int_{0}^{t} \mathcal{J}(t-s)\left(1+\sup _{(r, y) \in[0, s] \times \mathbb{R}^{d}} \mathbb{E}\left[\left|u^{\varepsilon}(r, y)\right|^{p}\right]\right) d s \\
& \leq \varepsilon c(p, L, T) .
\end{aligned}
$$

For the second term $A_{2}^{\varepsilon}$, by the Lipschitz property of $b$ and Fubini's theorem, we have

$$
\begin{aligned}
\mathbb{E}\left[\left|A_{2}^{\varepsilon}(t, x)\right|^{p}\right] & \leq c(p, T) \mathbb{E}\left[\int_{0}^{t} \int_{\mathbb{R}^{d}} \mathbf{G}_{\underline{\alpha}, \underline{\delta}}(t-s, x-y)\left|u^{\varepsilon}(s, y)-u^{0}(s, y)\right|^{p} d y d s\right] \\
& \leq c(p, L, T) \int_{0}^{t} \int_{\mathbb{R}^{d}} \mathbf{G}_{\underline{\alpha}, \underline{\delta}}(t-s, x-y) \mathbb{E}\left[\sup _{0 \leq l \leq s, z \in \mathbb{R}^{d}}\left|u^{\varepsilon}(l, z)-u^{0}(l, z)\right|^{p}\right] d y d s \\
& \leq c(p, L, T) \int_{0}^{t} \mathbb{E}\left[\sup _{0 \leq l \leq s, z \in \mathbb{R}^{d}}\left|u^{\varepsilon}(l, z)-u^{0}(l, z)\right|^{p}\right] d s .
\end{aligned}
$$

Set $\zeta^{\varepsilon}(t):=\sup _{0 \leq s \leq t, x \in \mathbb{R}^{d}} \mathbb{E}\left[\left|u^{\varepsilon}(s, x)-u^{0}(s, x)\right|^{p}\right]$. By (12), (14), and (15), we have that, for any $t \in[0, T]$,

$$
\zeta^{\varepsilon}(t) \leq \varepsilon^{\frac{p}{2}} c_{1}(p, L, T)+c_{2}(p, L, T) \int_{0}^{t} \zeta^{\varepsilon}(s) d s .
$$

Hence, by Gronwall's lemma, there exists a constant $c(p, L, T)$ independent of $\varepsilon$ such that

$$
\zeta^{\varepsilon}(T) \leq \varepsilon^{\frac{p}{2}} c(p, L, T)
$$

The proof is complete.

\section{Central limit theorem}

To study the central limit theorem for $u^{\varepsilon}$, we furthermore suppose that

(D): The function $b$ is differentiable, and its derivative $b^{\prime}$ is Lipschitz. More precisely, there exists a positive constant $L^{\prime}$ such that

$$
\left|b^{\prime}(y)-b^{\prime}(z)\right| \leq L^{\prime}|y-z| \quad \text { for all } y, z \in \mathbb{R} .
$$

Combined with the Lipschitz continuity of $b$, we conclude that

$$
\left|b^{\prime}(z)\right| \leq L, \quad \forall z \in \mathbb{R}
$$


Consider the stochastic partial differential equation

$$
\frac{\partial X}{\partial t}(t, x)=\mathcal{D}_{\underline{\delta}}^{\underline{\alpha}} X(t, x)+b^{\prime}\left(u^{0}(t, x)\right) X(t, x)+\sigma\left(u^{0}(t, x)\right) \dot{F}(t, x),
$$

with $X(0, x) \equiv 0$. Using the same strategy in the proof of the existence and uniqueness for the solution to Eq. (1), one can obtain the following result. Here we omit its proof.

Proposition 3.1 Assuming conditions $(\mathrm{C}),\left(H_{\eta}^{\alpha}\right)$, and (D), there exists a unique continuous solution $X$ to Eq. (18) in the following form: for any $t \in[0, T], x \in \mathbb{R}^{d}$,

$$
\begin{aligned}
X(t, x)= & \sum_{k \geq 1} \int_{0}^{t}\left\langle\mathbf{G}_{\underline{\alpha}, \underline{\delta}}(t-s, x-\cdot) \sigma\left(u^{0}(s, \star)\right), e_{k}\right\rangle_{\mathcal{H}} d B_{s}^{k} \\
& +\int_{0}^{t}\left[\mathbf{G}_{\underline{\alpha}, \underline{\delta}}(t-s) *\left[b^{\prime}\left(u^{0}(s, \star)\right) X(s, \star)\right]\right](x) d s .
\end{aligned}
$$

Our main result is the following central limit theorem.

Theorem 3.2 Assuming conditions $(\mathrm{C}),\left(H_{\eta}^{\alpha}\right)$, and $(\mathrm{D})$, for any $(t, x) \in[0, T] \times \mathbb{R}^{d}$, the process $\left(u^{\varepsilon}(t, x)-u^{0}(t, x)\right) / \sqrt{\varepsilon}$ converges in $L^{2}$ to a random field $X(t, x)$.

Proof Let

$$
X^{\varepsilon}=\left(u^{\varepsilon}(t, x)-u^{0}(t, x)\right) / \sqrt{\varepsilon} .
$$

Then, for any $(t, x) \in[0, T] \times \mathbb{R}^{d}$,

$$
\begin{aligned}
X^{\varepsilon}(t, x) & -X(t, x) \\
= & \sum_{k \geq 1} \int_{0}^{t}\left\langle\mathbf{G}_{\underline{\alpha}, \underline{\delta}}(t-s, x-\star)\left[\sigma\left(u^{\varepsilon}(s, \star)\right)-\sigma\left(u^{0}(s, \star)\right)\right], e_{k}\right\rangle_{\mathcal{H}} d B_{s}^{k} \\
& +\int_{0}^{t}\left\{\mathbf{G}_{\underline{\alpha}, \underline{\underline{\delta}}}(t-s) *\left[\frac{b\left(u^{\varepsilon}(s, \star)\right)-b\left(u^{0}(s, \star)\right)}{\sqrt{\varepsilon}}-b^{\prime}\left(u^{0}(s, \star)\right) X(s, \star)\right](x)\right\} d s \\
= & \sum_{k \geq 1} \int_{0}^{t}\left\{\mathbf{G}_{\underline{\alpha}, \underline{\delta}}(t-s, x-\star)\left[\sigma\left(u^{\varepsilon}(s, \star)\right)-\sigma\left(u^{0}(s, \star)\right)\right], e_{k}\right\rangle_{\mathcal{H}} d B_{s}^{k} \\
& +\int_{0}^{t}\left\{\mathbf{G}_{\underline{\alpha}, \underline{\delta}}(t-s) *\left[\frac{b\left(u^{\varepsilon}(s, \star)\right)-b\left(u^{0}(s, \star)\right)}{\sqrt{\varepsilon}}-b^{\prime}\left(u^{0}(s, \star)\right) X^{\varepsilon}(s, \star)\right](x)\right\} d s \\
& +\int_{0}^{t}\left\{\mathbf{G}_{\underline{\alpha}, \underline{\delta}}(t-s) *\left[b^{\prime}\left(u^{0}(s, \star)\right)\left(X^{\varepsilon}(s, \star)-X(s, \star)\right)\right](x)\right\} d s \\
=: & A_{1}^{\varepsilon}(t, x)+A_{2}^{\varepsilon}(t, x)+A_{3}^{\varepsilon}(t, x) .
\end{aligned}
$$

For the first term $A_{1}^{\varepsilon}$, by the Lipschitz continuity of $\sigma$ and Proposition 2.2, we have

$$
\begin{aligned}
\mathbb{E}\left[\left|A_{1}^{\varepsilon}(t, x)\right|^{2}\right] & =\mathbb{E}\left[\int_{0}^{t}\left\|\mathbf{G}_{\underline{\alpha}, \underline{\delta}}(t-s, x-\star)\left[\sigma\left(u^{\varepsilon}(s, \star)\right)-\sigma\left(u^{0}(s, \star)\right)\right]\right\|_{\mathcal{H}}^{2} d s\right] \\
& \leq L^{2} \mathbb{E}\left[\int_{0}^{t}\left\|\mathbf{G}_{\underline{\alpha}, \underline{\delta}}(t-s, x-\star)\left|u^{\varepsilon}(s, \star)-u^{0}(s, \star)\right|\right\|_{\mathcal{H}}^{2} d s\right]
\end{aligned}
$$




$$
\begin{aligned}
& \leq L^{2} \int_{0}^{t} d s\left(\sup _{(r, y) \in[0, s] \times \mathbb{R}^{d}} \mathbb{E}\left|u^{\varepsilon}(r, y)-u^{0}(r, y)\right|^{2}\right) \times \mathcal{J}(t-s) \\
& \leq \varepsilon c(L, T) .
\end{aligned}
$$

By Taylor's formula, there exists a random field $\eta^{\varepsilon}$ taking values in $(0,1)$ such that

$$
b\left(u^{\varepsilon}\right)-b\left(u^{0}\right)=b\left(u^{0}+\sqrt{\varepsilon} X^{\varepsilon}\right)-b\left(u^{0}\right)=\sqrt{\varepsilon} b^{\prime}\left(u^{0}+\sqrt{\varepsilon} \eta^{\varepsilon} X^{\varepsilon}\right) X^{\varepsilon} .
$$

Since $b^{\prime}$ is Lipschitz continuous, we have

$$
\left|\frac{1}{\sqrt{\varepsilon}}\left[b\left(u^{0}+\sqrt{\varepsilon} X^{\varepsilon}\right)-b\left(u^{0}\right)\right]-b^{\prime}\left(u^{0}\right) X^{\varepsilon}\right| \leq \sqrt{\varepsilon} L^{\prime}\left|X^{\varepsilon}\right|^{2} .
$$

Thus, by Proposition 2.2, we have

$$
\begin{aligned}
\mathbb{E}\left[\left|A_{2}^{\varepsilon}(t, x)\right|^{2}\right] & \leq \varepsilon L^{\prime 2} \mathbb{E}\left[\left(\int_{0}^{t} \mathbf{G}_{\underline{\alpha}, \underline{\delta}}(t-s) *\left|X^{\varepsilon}(s, \star)\right|^{2}(x) d s\right)^{2}\right] \\
& \leq \varepsilon c\left(L^{\prime}, T\right) \int_{0}^{t} \int_{\mathbb{R}^{d}} \mathbf{G}_{\underline{\alpha}, \underline{\delta}}(t-s, x-y) \mathbb{E}\left[\left|X^{\varepsilon}(s, y)\right|^{4}\right] d s d y \\
& \leq \varepsilon c\left(L^{\prime}, T\right) \int_{0}^{t} \int_{\mathbb{R}^{d}} \mathbf{G}_{\underline{\alpha}, \underline{\delta}}(t-s, x-y) \sup _{(r, z) \in[0, s] \times \mathbb{R}^{d}} \mathbb{E}\left[\left|X^{\varepsilon}(r, z)\right|^{4}\right] d s d y \\
& \leq \varepsilon c\left(L, L^{\prime}, T\right) .
\end{aligned}
$$

For the third term, by the boundedness of $b^{\prime}$, we have

$$
\begin{aligned}
\mathbb{E}\left[\left|A_{3}^{\varepsilon}(t, x)\right|^{2}\right] & \leq c(L, T) \mathbb{E}\left[\int_{0}^{t} \int_{\mathbb{R}^{d}} \mathbf{G}_{\underline{\alpha}, \underline{\delta}}(t-s, x-y)\left|X^{\varepsilon}(s, y)-X(s, y)\right|^{2}(x) d s d y\right] \\
& \leq c(L, T) \int_{0}^{t} \int_{\mathbb{R}^{d}} \mathbf{G}_{\underline{\alpha}, \underline{\delta}}(t-s, x-y) \sup _{(r, z) \in[0, s] \times \mathbb{R}^{d}} \mathbb{E}\left[\left|X^{\varepsilon}(r, z)-X(r, z)\right|^{2}\right] d s d y \\
& \leq c(L, T) \int_{0}^{t} \sup _{(r, z) \in[0, s] \times \mathbb{R}^{d}} \mathbb{E}\left[\left|X^{\varepsilon}(r, z)-X(r, z)\right|^{2}\right] d s .
\end{aligned}
$$

By (20)-(23), we have that, for any $t \in[0, T]$,

$$
\begin{aligned}
& \sup _{(r, x) \in[0, t] \times \mathbb{R}^{d}} \mathbb{E}\left[\left|X^{\varepsilon}(r, x)-X(r, x)\right|^{2}\right] \\
& \leq \varepsilon c\left(L, L^{\prime}, T\right)+\int_{0}^{t} \sup _{(r, z) \in[0, s] \times \mathbb{R}^{d}} \mathbb{E}\left[\left|X^{\varepsilon}(r, z)-X(r, z)\right|^{2}\right] d s .
\end{aligned}
$$

Hence, by Gronwall's lemma, there exists a constant $c\left(L, L^{\prime}, T\right)$ independent of $\varepsilon$ such that

$$
\sup _{(t, x) \in[0, T] \times \mathbb{R}^{d}} \mathbb{E}\left[\left|X^{\varepsilon}(t, x)-X(t, x)\right|^{2}\right] \leq \varepsilon c\left(L, L^{\prime}, T\right) .
$$

The proof is complete. 
Acknowledgements

The author is grateful to the anonymous referees for conscientious comments and corrections.

\section{Funding}

This research was financially supported by the Natural Science Foundation of China (11471304).

Availability of data and materials

Data sharing not applicable to this article as no data sets were generated or analyzed during the current study.

\section{Competing interests}

The author declares that they have no competing interests.

\section{Authors' contributions}

The author carried out the proofs of the main results and approved the final manuscript.

\section{Publisher's Note}

Springer Nature remains neutral with regard to jurisdictional claims in published maps and institutional affiliations.

Received: 5 December 2019 Accepted: 26 February 2020 Published online: 04 March 2020

\section{References}

1. Boulanba, L., Eddahbi, M., Mellouk, M.: Fractional SPDEs driven by spatially correlated noise: existence of the solution and smoothness of its density. Osaka J. Math. 47, 41-65 (2010)

2. Cheng, L., Li, R., Wang, R., Yao, N.: Moderate deviations for a stochastic wave equation in dimension three. Acta Appl. Math. 158, 67-85 (2018)

3. Da Prato, G., Zabczyk, J.: Stochastic Equations in Infinite Dimensions. Cambridge University Press, Cambridge (1992)

4. Dalang, R.: Extending the martingale measure stochastic integral with applications to spatially homogeneous s.p.d.e's. Electron. J. Probab. 4, 6, 1-29 (1999)

5. Dalang, R., Quer-Sardanyons, L.: Stochastic integrals for SPDE's: a comparison. Expo. Math. 29(1), 67-109 (2011)

6. Droniou, J., Imbert, C.: Fractal first-order partial differential equations. Arch. Ration. Mech. Anal. 182(2), 299-331 (2006)

7. El Mellali, T., Mellouk, M.: Large deviations for a fractional stochastic heat equation in spatial dimension $\mathbb{R}^{d}$ driven by a spatially correlated noise. Stoch. Dyn. 16, 1650001 (2015)

8. Freidlin, M.I., Wentzell, A.D.: Random Perturbation of Dynamical Systems. Springer, Berlin (1984). Translated by J. Szuc

9. Gao, F., Wang, S.: Asymptotic behaviors for functionals of random dynamical systems. Stoch. Anal. Appl. 34(2), $258-277$ (2016)

10. Komatsu, T:: On the martingale problem for generators of stable processes with perturbations. Osaka J. Math. 21(1), 113-132 (1984)

11. Li, Y., Wang, R., Yao, N., Zhang, S.: Moderate deviations for a fractional stochastic heat equation with spatially correlated noise. Stoch. Dyn. 17(4), 1750025 (2017)

12. Walsh, J.: An introduction to stochastic partial differential equations. In: École d'été de Probabilités St Flour XIV. Lect Notes Math., vol. 1180. Springer, Berlin (1986)

13. Wang, R., Zhang, T.: Moderate deviations for stochastic reaction-diffusion equations with multiplicative noise. Potential Anal. 42,99-113 (2015)

\section{Submit your manuscript to a SpringerOpen ${ }^{0}$ journal and benefit from:}

- Convenient online submission

$\checkmark$ Rigorous peer review

Open access: articles freely available online

- High visibility within the field

Retaining the copyright to your article

Submit your next manuscript at $\gg$ springeropen.com 